Published in final edited form as:

Cancer. 2018 February 01; 124(3): 521-529. doi:10.1002/cncr.30954.

\title{
Patient-Reported Quality of Life Outcomes after De-Escalated Chemoradiation for Human Papillomavirus-Positive Oropharyngeal Carcinoma: Findings from a Phase II Trial
}

\author{
John V. Hegde, MD1', Narek Shaverdian, MD², Megan E. Daly, MD², Carol Felix ${ }^{1}$, Deborah J. \\ Wong, MD, PhD ${ }^{3}$, Michael H. Rosove, MD $^{3}$, Jordan Garst ${ }^{1}$, Pin-Chieh Wang, PhD ${ }^{1}$, Darlene \\ Veruttipong, $\mathrm{MPH}^{1}$, Shyam Rao, MD, $\mathrm{PhD}^{2}$, Ruben C. Fragoso, MD, $\mathrm{PhD}^{2}$, Jonathan Riess, \\ MD4, Michael L. Steinberg, MD1, and Allen M. Chen, MD1 \\ ${ }^{1}$ Department of Radiation Oncology, University of California, Los Angeles-David Geffen School of \\ Medicine, Los Angeles, CA, 90095 \\ ${ }^{2}$ Department of Radiation Oncology, University of California, Davis, School of Medicine, \\ Sacramento, CA 95817 \\ ${ }^{3}$ Division of Hematology and Oncology, Department of Internal Medicine, University of California, \\ Los Angeles-David Geffen School of Medicine, Los Angeles, CA, 90095 \\ ${ }^{4}$ Division of Hematology and Oncology, Department of Internal Medicine, University of California, \\ Davis, School of Medicine, Sacramento, CA 95817
}

\begin{abstract}
Purpose-This study represents a subset analysis of quality of life outcomes among patients treated on a phase II trial of de-escalated chemoradiation for human papillomavirus (HPV)associated oropharynx cancer.

Methods-Eligibility included newly-diagnosed, stage III or IV oropharyngeal squamous cell carcinoma, p16-positivity, age at least 18 years, and Zubrod performance status $0-1$. Treatment was induction paclitaxel $175 \mathrm{mg} / \mathrm{m}^{2}$ and carboplatin AUC 6 for 2 cycles followed by responseadapted, dose-reduced radiation of $54 \mathrm{~Gy}$ or $60 \mathrm{~Gy}$ with weekly concurrent paclitaxel $30 \mathrm{mg} / \mathrm{m}^{2}$. The University of Washington Quality of Life (UW-QOL) and the Functional Assessment of
\end{abstract}

Corresponding Author: Allen M. Chen, MD, Department of Radiation Oncology, University of Kansas School of Medicine, Mail Stop 4033, 3901 Rainbow Blvd., Kansas City, KS 66160, Phone: 913-588-3612; Fax: 913-588-3663, achen5@kumc.edu.

Disclosures: The authors report no conflicts of interest

Author contributions: JH: Conceptualization, methodology, validation, formal analysis, investigation, data curation, visualization, writing-original draft, and writing-review and editing. NS: Validation, formal analysis, investigation, data curation, writing-review and editing. MD: Methodology, validation, formal analysis, investigation, data curation, supervision, visualization, writing-review and editing. CF: Resources, data curation, writing-review and editing. DW: investigation, data curation, writing-review and editing. MR: investigation, data curation, writing-review and editing. JG: Software, resources, data curation, project administration, writing-review and editing. PW: Software, resources, data curation, project administration, writing-review and editing. DV: Software, resources, data curation, project administration, writing-review and editing. SR: investigation, data curation, writing-review and editing. RF: investigation, data curation, writing-review and editing. JR: Investigation, data curation, writing-review and editing. MS: Resources, writing-review and editing, supervision, project administration. AC: Conceptualization, methodology, validation, formal analysis, investigation, resources, data curation, visualization, writing-original draft, writing-review and editing, supervision, project administration, funding acquisition. 
Cancer Therapy-Head and Neck (FACT-H\&N) questionnaires were used to assess patient-reported quality of life as a secondary endpoint.

Results-Forty-five patients were registered, of whom 40 completed quality of life surveys and were evaluable. Nadirs for overall UW-QOL and FACT-H\&N scores were reached at 4 weeks after treatment but returned to baseline at 3 months. Nearly all functional indices returned to baseline levels by 6 to 9 months. The mean overall UW-QOL score was 71.6 at baseline compared to 70.8 , 73.0, 83.3, and 81.1, at 3-months, 6-months, 1-year, and 2-years, respectively, post-therapy. The proportion rating their overall quality of life as "very good" or "outstanding" at 6-months, 1-year, and 2-years with the UW-QOL was 50\%, 77\%, and 84\%, respectively.

Conclusion-This de-escalation regimen achieved quality of life outcomes that were favorable compared to historical controls. These results serve as powerful evidence that ongoing deescalation efforts lead to tangible gains in function and quality of life.

\section{Keywords}

de-escalation; chemoradiation; HPV; oropharyngeal; quality of life; head and neck

\section{Introduction}

While concurrent chemoradiation represents a potentially curative therapeutic option for locally advanced squamous cell carcinoma of the head and neck, it has historically been associated with significant long-term toxicity $(1,2)$. Common side effects include swallowing dysfunction, fatigue, xerostomia, and salivary changes, among others. Given the increasing recognition of the influence that quality of life has on survivorship for patients with head and neck cancer, incorporating this endpoint in the evaluation of efficacy is becoming more important to refine treatment strategies (3). This is particularly relevant as survival rates continue to improve for this disease and patients are living longer. Although modern techniques with intensity-modulated radiotherapy (IMRT) have been associated with improvements in patient-reported quality of life, the incidence of treatment-related morbidity remains high (4).

For example, the dose threshold to cause dysphagia due to pharyngeal constrictor muscle or larynx dysfunction likely rests at or below $60 \mathrm{~Gy}$ (5-8). This threshold may be challenging to avoid in some clinical scenarios when gross disease rests immediately adjacent to these structures and the maximum radiation dose level is $70 \mathrm{~Gy}$. In addition, multiple studies have noted that even with modern treatment techniques, quality of life recovery often takes more than 1 year (9-12). As such, lower maximum dose levels may improve the morbidity profile of treatment.

It is now well-established that patients with oropharyngeal squamous cell carcinoma related to the human papillomavirus (HPV) have both a more robust response to treatment and an improved overall prognosis compared to those whose tumors are not HPV-related (13-15). Due to an abundance of pre-clinical and clinical data which has demonstrated that HPVpositive tumors are exquisitely radiosensitive, interest has arisen focusing on the attenuation of treatment regimens with the aim of improving quality of life while maintaining rates of 
disease control (16-18). We have previously reported the results of a phase II de-escalation trial evaluating the use of reduced doses of radiation and demonstrated excellent rates of progression-free and overall survival (19). The aim of this follow-up report is to document trends in quality of life for patients treated on this prospective clinical trial.

\section{Methods and Materials}

From October 2012 to March 2015, a total of 45 patients with HPV-positive oropharyngeal carcinoma were enrolled on a phase II trial of de-escalated chemoradiation conducted jointly between the University of California, Davis and the University of California, Los Angeles, Schools of Medicine. This subset analysis was designed to report on the secondary endpoint of quality of life and was comprised of 40 subjects that completed quality of life datasets and who were without evidence of disease at most recent follow-up. Eligible participants were 18 years of age or older with Zubrod performance status of $0-1$, and a histological diagnosis of newly diagnosed, stage III or IV, HPV-associated squamous cell carcinoma arising from the oropharynx. Smoking history was not used as a criterion for eligibility. HPV-positivity was defined as tumors that were p16-positive by immunohistochemistry. Baseline patient characteristics are reported in Table 1. The protocol of the study was approved by the Institutional Review Boards of both universities, and all study participants provided written informed consent. The trial is registered with clinicaltrials.gov (NCT02048020 and NCT01716195) and opened separately at the University of California, Davis and the University of California, Los Angeles, Schools of Medicine, respectively, before the data was centrally combined for aggregate analysis.

Two cycles of induction chemotherapy (paclitaxel $175 \mathrm{mg} / \mathrm{m}^{2}$ infused over 3 hours followed by carboplatin $\mathrm{AUC}=6$ as a 30 minute infusion) were administered to all patients primarily as a means to select HPV-positive oropharyngeal carcinoma patients, who may benefit from significant radiation dose de-escalation. At least 2 weeks after completion of induction chemotherapy, concurrent chemoradiation was initiated using single-agent paclitaxel infused at a dose of $30 \mathrm{mg} / \mathrm{m}^{2}$ over at least 1 hour given weekly with daily radiation for 5 total cycles. The radiation dose was determined by the clinical response to induction chemotherapy using RECIST criteria (20). For patients with a complete response or partial response, the total prescribed dose to the primary tumor and involved nodes were $54 \mathrm{~Gy}$ in 27 fractions. All other patients were classified as having minor response and received $60 \mathrm{~Gy}$ in 30 fractions. IMRT was mandated.

Quality of life assessment was performed using 2 previously validated surveys administered at registration and with each follow-up visit. Patients were asked to complete these instruments in paper form in a private setting with the assistance of nursing staff if necessary. The University of Washington Quality of Life Scale (UW-QOL), version 4, is a survey used to evaluate patient-reported quality of life outcomes in head and neck cancer (21). The UW-QOL consists of 12 domains pertaining to quality of life in the categories of pain, appearance, activity, recreation, swallowing, chewing, speech, shoulder function, taste, saliva, mood, and anxiety. A score of 0 indicates very poor or no functional capacity with regard to that domain while a score of 100 indicates no disability in that domain. In the final part of the UW-QOL, patients were asked general questions focused on quality of life. This 
segment was scored with 0 indicating very poor quality of life and 100 indicating outstanding quality of life, with a range of scores as integer values between. The Functional Assessment of Cancer Therapy-Head and Neck Scale (FACT-H\&N) is a validated, multidimensional, self-reported quality of life instrument specifically designed for use with head and neck cancer patients (22). It consists of 27 core items which assess patient function in four domains: Physical, Social/Family, Emotional, and Functional well-being, which is further supplemented by 12 site-specific items to assess for head and neck-related symptoms. Each item is rated on a 0 to 4 Likert-type scale (with higher scores representing better quality of life), and then combined to produce subscale scores for each domain, as well as a global quality of life score. The FACT-G sub-score (encompassing physical, social, emotional, and function well-being sub-scales) and FACT-HN sub-score (encompassing the head and neck-specific domain alone) were also determined. Quality of life data was presented using descriptive statistics. Any patient with a missing survey was excluded in the data set for that particular time point. Differences in proportions among subsets was evaluated using the Fisher's exact test. A two-tailed t-test was used to compare quality of life scores at time points following treatment with baseline values. Logistical regression was performed to evaluate whether pre-treatment or treatment factors, including increasing age, smoking history ( $\leq 10$ vs. $>10$ pack years), alcohol history (none to moderate vs. heavy), gender, T-classification, $\mathrm{N}$-classification, site of primary, and radiation dose were associated with UW-QOL and FACT-H\&N scores at both 6 and 12 months post-treatment. A p-value of 0.05 was set for statistical significance.

\section{Results UW-QOL analysis}

The baseline, 6-month, 12-month, 18-month, and 24-month post-treatment UW-QOL survey completion rates were 90\%, 53\%, 75\%, and 50\%. Table S1 (Supporting Information) outlines UW-QOL scores for overall, health-related, and function-related quality of life at various time points relative to baseline. The mean overall and health-related quality of life scores as determined by the UW-QOL were 71.6 (range 40-100) and 69.7 (range 20-100) at baseline. As illustrated in Figure 1, the nadirs were reached at approximately 4 weeks posttreatment for both measures. Table S2 (Supporting Information) outlines the percentage change in each of the UW-QOL domains from baseline values. The mean overall quality of life values were 70.8, 73.0, 83.3, and 81.1 at 3 months, 6 months, 1 year, and 2 years, respectively, after completion of treatment. At 3 months, twelve of 37 patients (32\%) continued to experience a decline in their overall quality of life scores compared to baseline. However, no significant decrease in the mean overall quality of life score compared to baseline existed at 3 months ( 70.8 vs. $71.6, \mathrm{p}=0.86$ ) or any time point thereafter. By 18 months post-therapy, the mean overall quality of life score (81.6) actually exceeded the value at baseline $(\mathrm{p}=0.002)$. At 6 months, 1 year, and 2 years post-treatment, the proportion of patients reporting a decline from baseline was $40 \%, 13 \%$, and $32 \%$, respectively. For health-related quality of life, the mean scores at 3 months, 6 months, 1 year, and 2 years, respectively, were $63.2,71.0,79.3$, and 74.7. The corresponding proportion of patients reporting a decline in their health-related quality of life compared to baseline was $38 \%$, $30 \%, 27 \%$, and $26 \%$, respectively. 
Figure 2 illustrates the individual domain scores for the UW-QOL survey over time. Domain scores for taste $(\mathrm{p}<0.0001)$ and salivary function $(\mathrm{p}<0.0001)$ remained approximately $49 \%$ and $51 \%$ lower in magnitude, respectively, compared to baseline when assessed at 6 months post-treatment (Tables S1-S2). Median quality of life scores related to swallowing returned to baseline at 15 months $(\mathrm{p}=0.28)$. While taste and salivary function were more sluggish to recover, continued improvement was observed even from the 18 month to 24 month time points. The composite UW-QOL score, averaging all 12 domains, returned to baseline at approximately 9 months post-treatment $(\mathrm{p}=0.38)$.

By 3 months, there was no difference in how patients rated their health-related $(\mathrm{p}=0.11)$ and overall quality of life $(\mathrm{p}=0.86)$ over the past 7 days compared to baseline for both. The proportion rating their overall quality of life as "very good" or "outstanding" at 6 months, 1 year, and 2 years was $50 \%, 77 \%$, and $84 \%$, respectively. The proportion rating their overall quality of life as "good" during these time points were $25 \%, 17 \%$, and $11 \%$, respectively. With respect to health-related quality of life, $50 \%, 77 \%$, and $63 \%$ rated this as "very good" or "outstanding" at 6 months, 1 years, and 2 years, respectively.

\section{FACT-H\&N analysis}

The baseline, 6-month, 12-month, 18-month, and 24-month post-treatment FACT-H\&N survey completion rates were $78 \%, 40 \%, 43 \%$, and $35 \%$. The FACT-H\&N quality of life survey results are outlined in Table 2. The mean total score, G sub-score, and HN sub-score from the FACT-H\&N was 120.4, 88.3, and 32.1, respectively, at baseline. At 4 weeks after completion of protocol therapy, the corresponding values were 97.0, 77.1, and 19.9, each of which were significantly depressed from baseline and representing the nadirs during the post-treatment period ( $\mathrm{p}<0.05$, for all). However, at 3 months post-treatment, the mean total score and FACT-G sub-score was 112.3 and 87.4, respectively, which represented statistical returns to baseline values ( $\mathrm{p}>0.05$, for both). The mean FACT-HN sub-score was 24.9 which was still significantly lower than its baseline value $(\mathrm{p}<0.001)$. At approximately, 6 months, the mean total score and FACT-G sub-score was 122.0 and 97.0, respectively, which did not differ significantly from baseline ( $p>0.05$, for both). The mean FACT-HN sub-score was 27.4 at this time point which continued to be significantly lower compared to baseline $(\mathrm{p}=0.0012)$. At 9 months, the mean FACT-HN sub-score had increased to 26.1 which did not differ significantly from baseline for the evaluable patients $(\mathrm{p}=0.099)$. The mean total score, FACT-G sub-score, and FACT-HN sub-score was 126.5, 95.0, and 31.4, respectively, at 12 months. The mean total score, FACT-G sub-score, and FACT-HN sub-score was 123.0, 93.6, and 29.4, respectively, at 24 months.

\section{Factors impacting quality of life}

On univariate analysis, none of the pre-treatment or treatment-related factors were predictive for overall quality of life at 6 or 12 months by either reporting instrument ( $p>0.05$, for both). Trends were identified suggesting that base of tongue primary site predicted for worse UWQOL swallowing domain at 12 months (OR: 3.84; 95\% CI: 0.83-1.7.71, p=0.08) and that advancing age predicted for worse FACT-HN sub-scale score at 6 months (OR: 1.18; 95\% CI: $0.99-1.41, p=0.07)$. None of the other analyzed factors were associated with the UW- 
QOL scores related to health-related quality of life or any of the functional domains as well as the FACT-HN and FACT-G sub-scale scores at 6, 12, or 24 months ( $p>0.05$, for all).

As shown in Figure 3, no difference was observed between patients stratified to the 54 Gy and $60 \mathrm{~Gy}$ arms at any time point with respect to the overall UW-QOL score except at 4 weeks where the latter cohort had a significantly lower nadir in quality of life than the former (66.7 vs. 44.6, $\mathrm{p}=0.04)$. FACT-H\&N scores paralleled these findings with a differential nadir between the $54 \mathrm{~Gy}$ and $60 \mathrm{~Gy}$ arms significantly observed at 4 weeks (104.0 vs. 87.1, $\mathrm{p}=0.03$ ).

\section{Discussion}

This analysis of prospectively-acquired quality of life outcome data is the first to our knowledge to illustrate how reduced doses of radiation might influence survivorship for patients with locally advanced, HPV-positive oropharyngeal carcinoma treated by chemoradiation. Our findings provide powerful evidence that de-escalation strategies can indeed translate to tangible benefits for this population with respect to both physical and psychosocial functioning--leading to improvements in quality of life compared to historical controls. These results serve as validation for approaches investigating de-escalation and attest to the feasibility of enhancing quality of life for those historically treated with regimens associated with significant toxicity.

Most prominently, our findings demonstrating a return to baseline as early as 3 months after completion of de-escalated chemoradiation for many of the quality of life indices analyzed strongly suggest that functional recovery occurs more briskly and robustly than typically observed for patients treated with conventional regimens. As importantly, overall quality of life, as determined by both the UW-QOL and FACT-H\&N instruments, was maintained thereafter at baseline levels through 2 years post-treatment. These data strongly suggest that de-escalated chemoradiation accomplished its goal of reducing treatment-related morbidity and preserving long-term quality of life in patients with HPV-associated oropharyngeal carcinoma.

While nadirs in subjective functioning generally were reached at 4 weeks post-therapy, a gradual return to baseline in all domains was observed as expected. These were largely consistent with data published on patients treated with full-dose radiation regimens with the caveat that recovery clearly occurred earlier $(9-12,23)$. Surprisingly, mood and anxiety stabilized to levels above baseline even by time points within the acute toxicity phase, suggesting that the psychological consequences of treatment were not as profound as those seen typically for patients treated with conventional regimens. For instance, in a longitudinal study, Chen et al found that $85 \%$ of patients undergoing full-dose radiation experienced significant increases in self-reported depressive symptoms from prior to beginning radiation to completion (24).

The most encouraging finding from our analysis was that overall UW-QOL and FACT-H\&N scores returned nearly to baseline just 3 months following treatment for essentially all patients. The most direct comparison is to data from Vainshtein et al showing that most 
patients treated to standard radiation doses did not experience quality of life recovery until at least 1-year post-treatment (9). In a prospective study of 111 patients treated with IMRT for head and neck cancer, Tribius et al also showed that quality of life took 1 year to recover on average and that residual deficits persisted even at that point in the majority of subjects (10). Recently published subset analysis of large prospective trials conducted by the Radiation Therapy Oncology Group (RTOG) by Xiao et al and Truong et al evaluating patients treated by chemoradiation to 70 Gy similarly showed that HPV-associated patients experienced precipitous declines in quality of life with return to pre-treatment baseline not occurring until at least 1 year post-therapy in most patients $(11,12)$. Indeed, results from RTOG 0522 using a concurrent chemoradiation backbone of cisplatin with $70 \mathrm{~Gy}$ with or without cetuximab showed that at 1 year, continued differences in FACT-H\&N scores persisted for all subjects, which was a stark contrast from our findings among patients treated by deescalation (12). Ringash et al similarly showed that quality of life as measured by the FACT$\mathrm{H} \& \mathrm{~N}$ are sluggish to recover among patients treated by chemoradiation on a prospective trial conducted by the Trans-Tasmanian Oncology Group (23). Chen et al also reported an overall UW-QOL score of 67.5 at 1 year post-therapy among 84 patients treated by IMRT to standard doses, representing a $23 \%$ lower score than reported in the present series (4). Notably in that series, a significantly greater proportion of patients had yet to return to their quality of life baselines compared to the subjects in the present study.

Of course, not all subjects returned to their baseline overall quality of life score, with $32 \%$ reporting a decline from baseline at 2 years according to the UW-QOL. This may be related to several domains in the UW-QOL never returning to baseline following treatment. For example, the domain scores for taste $(\mathrm{p}<0.0001)$ and salivary function $(\mathrm{p}<0.0001)$ remain significantly lower than baseline 2 years following treatment, although continued improvement was seen in both from the 18- to 24-month time points. While the swallowing domain initially recovered, it subsequently began to decline slightly at 24 months. Therefore, although overall quality of life metrics appear very encouraging with dose de-escalated chemoradiation, it is worth noting that speech-and-swallow-related domains may still be affected long-term. Notably, all of these subjects participated in a clinical trial for which the main interest in participation was for the potential of improved salivary and swallowing function long-term from radiation dose de-escalation. As such, any long-term change in the function of these domains, however small, may have been more unfavorably seen by the study population as compared to patients treated with full-dose chemoradiation because of differing expectations.

The rationale for de-escalation lies in the assumption that relationships exist between increasing radiation dose to certain anatomical organs and the probability of clinical toxicity. Notably, the dose-response threshold (i.e. the steep part of the sigmoidal curve) for many side effects rests between 50 to $70 \mathrm{~Gy}$, suggesting that shifting a patient from one side of the normal tissue complication probability curve slope to the other will theoretically improve quality of life. For instance, data has shown that dose to such structures as the pharyngeal constrictor muscles, cricopharyngeal inlet, and larynx is of critical importance in predicting toxicity from chemoradiation and that the threshold for radiation-induced long-term dysphagia likely exists at approximately 55 to $60 \mathrm{~Gy}(5-8)$. The results of de-escalation efforts such as ours are thus particularly relevant because it is well-established that 
concurrent chemoradiation for head and neck cancer has eclipsed the limits of acceptable long-term toxicity. As importantly, our data demonstrating improvements in quality of life among patients with HPV-positive oropharyngeal cancer provide important reassurances legitimizing the premise of de-escalation.

Although direct comparisons are potentially confounded by differences in patient- and disease-characteristics, it is nonetheless apparent that the reported quality of life outcomes are amongst the most encouraging of any group of patients treated for head and neck cancer ever observed. However, we acknowledge that quantifying quality of life is difficult and can be complicated by non-treatment-related factors, including socioeconomic status, cultural/ spiritual influences, and medical comorbidities. Other limitations of our analysis relate to its relatively small sample size which precluded any meaningful subset analysis. The relatively short follow-up period of this study, coupled with the relatively low survey response rates at the longest follow-up period of 24 months (50\% for the UW-QOL and 35\% for the FACTHN surveys), are two further limitations. Given that some factors related to long-term quality of life, including dysphagia, may manifest many years following treatment, longer follow-up for a greater proportion of patients in this study will be helpful to more fully evaluate this treatment strategy. Moreover, this study did not have a control arm receiving full-dose chemoradiation. Therefore, comparisons of patient-reported outcomes between the conventional regimen and the two dose de-escalated arms in this study were unable to be performed. As a result, interpretation of the study data was limited to indirect comparison with previous studies of full-dose chemoradiation, resulting in the potential for biases inherent from comparisons with historical data. Additionally, it has been established that discrepancies can exist between patient- and physician-reported quality of life outcomes (25). Lastly, the possibility of participant bias needs to be acknowledged in that subjects who enrolled on this clinical trial may have possessed certain traits which predispose them to improved quality of life. It is worth noting that survey completion waned over time. This was likely due to inconsistent delivery of the instruments by study staff, insufficient time for completion at the visit, inconsistent adherence to follow-up encounters, and patient refusal, especially given the relatively long length of both surveys.

In conclusion, our findings provide the first evidence to our knowledge demonstrating that de-escalated chemoradiation for HPV-associated oropharyngeal carcinoma leads to improved quality of life outcomes compared to historical controls treated by more intensive, regimens using standard radiation doses. While our findings largely demonstrate that the use of reduced doses of radiation for patients with HPV-associated oropharyngeal carcinoma indeed translates into tangible benefits with respect to functioning and quality of life, they will require validation from other ongoing de-escalation trials.

\section{Supplementary Material}

Refer to Web version on PubMed Central for supplementary material.

\section{Acknowledgments}

Funding: University of California 
The authors thank Ms. Courtney Fisher for her assistance with manuscript preparation, formatting, and editing.

\section{References}

1. Hunter KU, Schipper M, Feng FY, et al. Toxicities affecting quality of life after chemo-IMRT of oropharyngeal cancer: propsective study of patient-reported, observer-rated, and objective outcomes. Int J Radiat Oncol Biol Phys. 2013; 85:935-940. [PubMed: 23040224]

2. Ward MC, Ross RB, Koyfman SA, et al. Modern image-guided intensity-modulated radiotherapy for oropharyx cancer and severe late toxic effects: implications for clinical trial design. JAMA Otolaryngol Head Neck Surg. 2016; 142:1164-1170. [PubMed: 27438230]

3. Chen AM, Daly ME, Farwell DG, et al. Quality of life among long-term survivors of head and neck cancer treated by intensity-modulated radiotherapy. JAMA Otolaryngol Head Neck Surg. 2014; 140:129-133. [PubMed: 24337483]

4. Chen AM, Farwell DG, Luu Q, et al. Intensity-modulated radiotherapy is associated with improved global quality of life among long-term survivors of head and neck cancer. Int J Radiat Oncol Biol Phys. 2012; 84:170-175. [PubMed: 22300572]

5. Feng FY, Kim HM, Lyden, et al. Intensity-modulated radiotherapy of head and neck cancer aiming to reduce dysphagia: early dose-effect relationships for the swallowing structures. Int J Radiat Oncol Biol Phys. 2007; 68:1289-1298. [PubMed: 17560051]

6. Deasy JO, Moiseenko V, Marks L, et al. Radiotherapy dose-volume effects on salivary gland function. Int J Radiat Oncol Biol Phys. 2010; 76(S):58-63.

7. Caudell JJ, Schaner PE, Desmond RA, et al. Dosimetric factors associated with long-term dysphagia after definitive radiotherapy for squamous cell carcinoma of the head and neck. Int J Radiat Oncol Biol Phys. 2010; 76:403-409. [PubMed: 19467801]

8. Li B, Li D, Lau DH, et al. Clinical-dosimetric analysis of measures of dysphagia including gastrostomy-tube dependence among head and neck cancer patients treated definitively by intensitymodulated radiotherapy with concurrent chemotherapy. Radiat Oncol. 2009; 4:52. [PubMed: 19909531]

9. Vainshtein JM, Moon DH, Feng FY, et al. Long-term quality of life after swallowing and salivarysparing chemo-intensity modulated radiation therapy in survivors of human papillomavirus-related oropharyngeal cancer. Int J Radiat Oncol Biol Phys. 2015; 91:925-33. [PubMed: 25832685]

10. Tribius S, Raguse M, Voigt C, et al. Residual deficits in quality of life one year after intensitymodulated radiotherapy for patients with locally advanced head and neck cancer: Results of a prospective study. Strahlenther Onkol. 2015; 191:501-510. [PubMed: 25747264]

11. Xiao C, Zhang Q, Nguyen-Tan PF, et al. Quality of life and performance status from a substudy conducted within a prospective phase 3 randomized trial of concurrent standard radiation versus accelerated radiation plus cisplatin for locally advanced head and neck carcinoma: NRG Oncology RTOG 0129. Int J Radiat Oncol Biol Phys. 2017; 97:667-677. [PubMed: 27727063]

12. Truong MT, Zhang Q, Rosenthal DI, et al. Quality of life and performance status from a substudy conducted within a prospective phase 3 randomized trial of concurrent standard radiation versus accelerated radiation plus cisplatin with or without cetuximab for locally advanced head and neck carcinoma: NRG Oncology RTOG 0522. Int J Radiat Oncol Biol Phys. 2016; 97:687-699. [PubMed: 27727066]

13. Rischin D, Young RJ, Fisher R, et al. Prognostic significance of p16INK4A and human papillomavirus in patients with oropharyngeal cancer treated on TROG 02.02 phase III trial. J Clin Oncol. 2010; 28:4142-8. [PubMed: 20697079]

14. Ang KK, Harris J, Wheeler R, et al. Human papillomavirus and survival of patients with oropharyngeal cancer. N Engl J Med. 2010; 363:24-35. [PubMed: 20530316]

15. Fakhry C, Westra WH, Li S, et al. Improved survival of patients with human papillomaviruspositive head and neck squamous cell carcinoma in a prospective clinical trial. J Natl Cancer Inst. 2008; 100:261-269. [PubMed: 18270337]

16. Gupta AK, Lee JH, Wilke WW, et al. Radiation response in two HPV-infected head-and-neck cancer cell lines in comparison to a non-HPV infected cell line and relationship to signaling through AKT. Int J Radiat Oncol Biol Phys. 2009; 74:928-933. [PubMed: 19480971] 
17. Chen AM, Li J, Beckett LA, et al. Differential response rates to irradiation among patients with human papillomavirus positive and negative oropharyngeal cancer. Laryngoscope. 2013; 123:152157. [PubMed: 23008061]

18. Vlashi E, Chen AM, Boyrie S, et al. Radiation-induced dedifferentiation of head and neck cancer cells into cancer stem cells depends on human papillomavirus. Int J Radiat Oncol Biol Phys. 2016; 94:1198-1206. [PubMed: 27026319]

19. Chen AM, Felix C, Wang PC, et al. Reduced-dose radiotherapy for human papillomavirusassociated squamous cell carcinoma of the oropharynx: a single-arm, phase 2 study. Lancet Oncol. 2017; 18:803-811. [PubMed: 28434660]

20. Eisenhauer EA, Therasse P, Bogaerts J, et al. New response evaluation criteria in solid tumors: Revised RECIST guideline (version 1.1). Eur J Cancer. 2009; 45:228-247. [PubMed: 19097774]

21. Hassan SJ, Weymuller EA. Assessment of quality of life in head and neck cancer patients. Head Neck. 1993; 15:485-496. [PubMed: 8253555]

22. Cella DF, Tulsky DS, Gray G, et al. The Functional Assessment of Cancer Therapy scale: development and validation of the general measure. J Clin Oncol. 1993; 11:570-9. [PubMed: 8445433]

23. Ringash J, Fisher R, Peters L, et al. Effect of p16 status on the quality-of-life experience during chemoradiation for locally advanced oropharyngeal cancer: A substudy of randomized trial TransTasman Radiation Oncology Group (TROG) 02. 02 (HeadSTART). Int J Radiat Oncol Biol Phys. 2017; 97:678-686. [PubMed: 27209505]

24. Chen AM, Jennelle RL, Grady V, et al. Prospective study of psychosocial distress among patients undergoing radiotherapy for head and neck cancer. Int J Radiat Oncol Biol Phys. 2009; 73:187193. [PubMed: 18513884]

25. Falchook AD, Green R, Knowles ME, et al. Comparison of patient- and practitioner-reported toxic effects associated with chemoradiotherapy for head and neck cancer. JAMA Otolaryngol Head Neck Surg. 2016; 142:517-523. [PubMed: 27149571] 


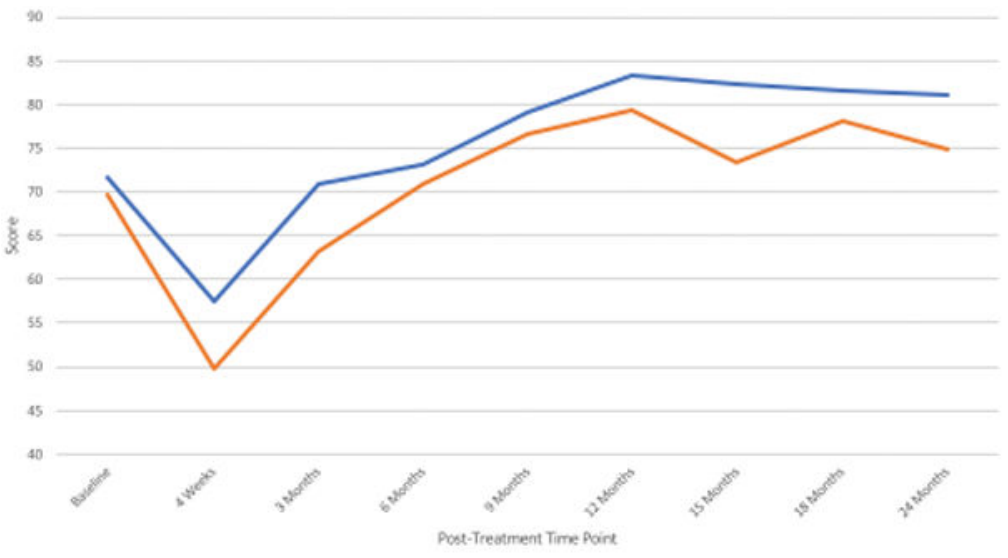

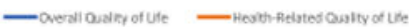

Figure 1.

Mean overall and health-related quality of life as determined by the UW-QOL for the entire subject population over time 
(A)

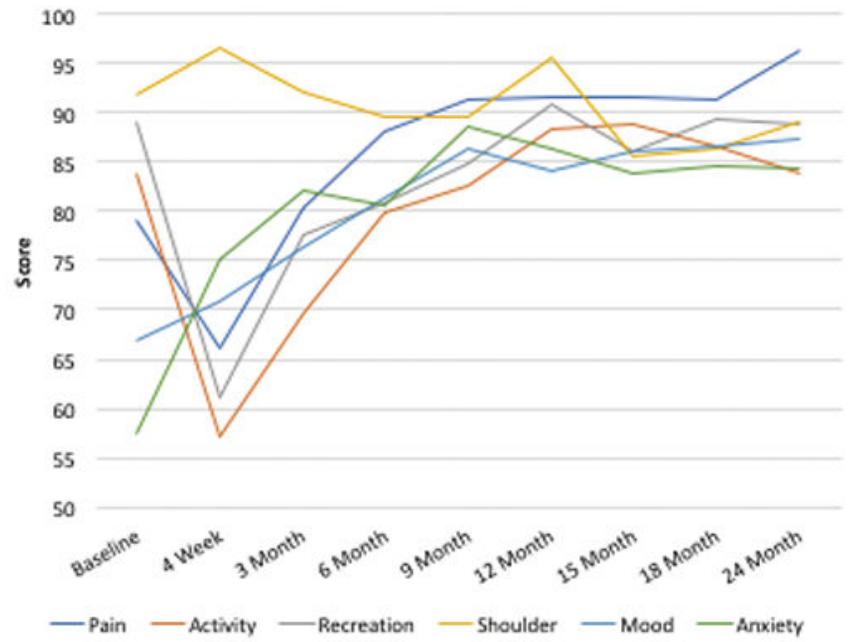

(B)

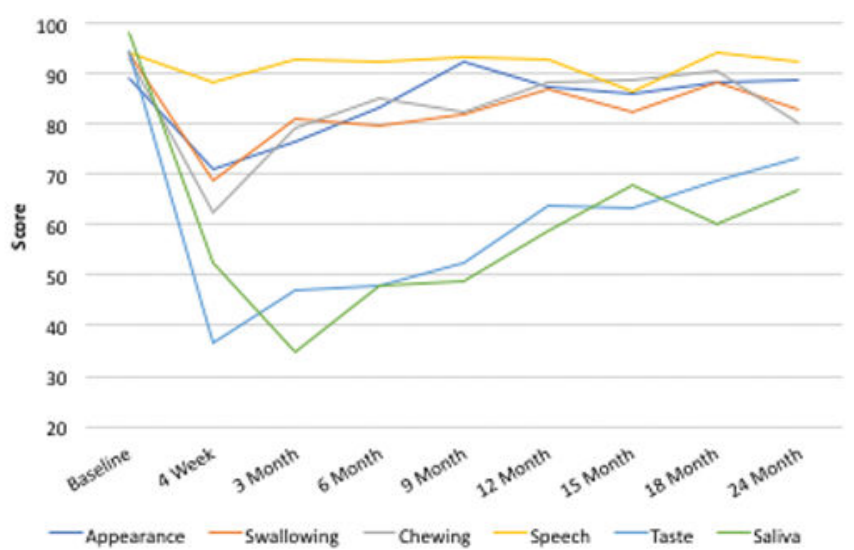

Figure 2.

Individual UW-QOL domain scores for the social-emotional (A) and physical (B) sub-scales for the entire subject population over time 
(A): Overall UW-QOL

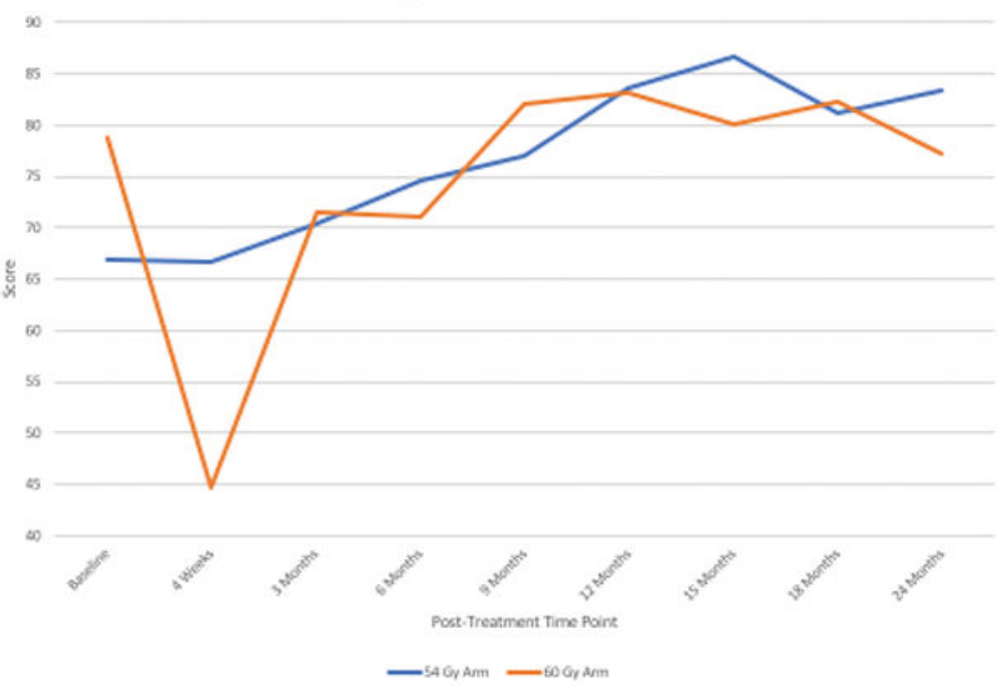

(B): Overall FACT-H\&N

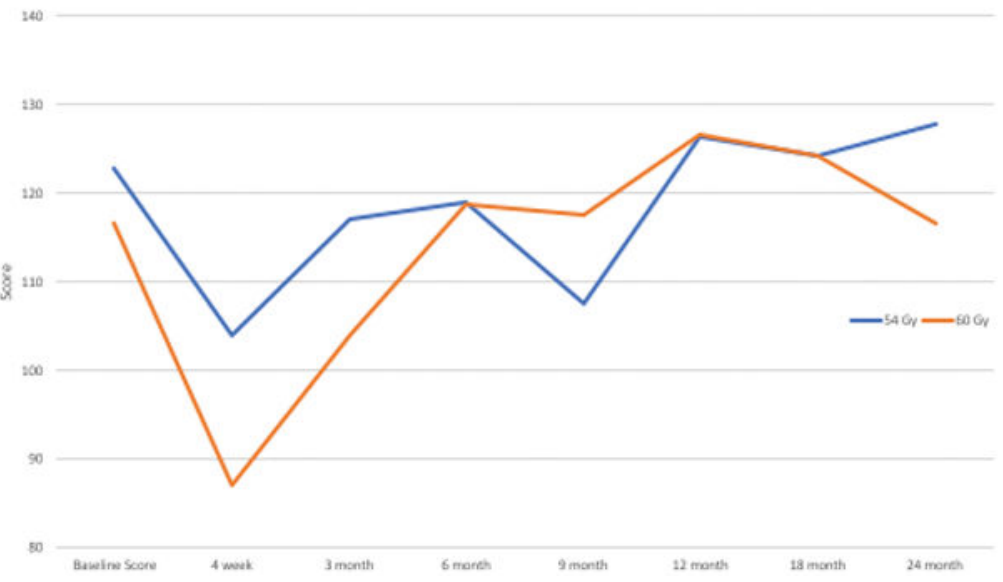

Figure 3.

Mean overall (A) UW-QOL and (B) FACT-H\&N scores, stratified by radiation dose 


\section{Table 1}

\begin{tabular}{|c|c|c|}
\hline \multirow{6}{*}{ 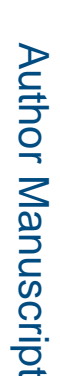 } & \multicolumn{2}{|c|}{ Baseline Patient Characteristics } \\
\hline & Age at Enrollment, years (SD) & $60.3(8.5)$ \\
\hline & Gender & \\
\hline & Male & $35(87.5 \%)$ \\
\hline & Female & $5(12.5 \%)$ \\
\hline & Ethnicity & \\
\hline \multirow{11}{*}{ 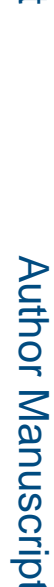 } & Non-Hispanic & $36(90.0 \%)$ \\
\hline & Hispanic & $3(7.5 \%)$ \\
\hline & African American & $1(2.5 \%)$ \\
\hline & Marital status & \\
\hline & Single & $6(15.0 \%)$ \\
\hline & Married & $31(77.5 \%)$ \\
\hline & Divorced & $2(5.0 \%)$ \\
\hline & Unknown & $1(2.5 \%)$ \\
\hline & Smoking history & \\
\hline & None & $21(52.5 \%)$ \\
\hline & $<10$ pack years & $10(25.0 \%)$ \\
\hline \multirow{11}{*}{ 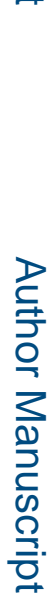 } & 10-30 pack years & $4(10.0 \%)$ \\
\hline & $>30$ pack years & $5(12.5 \%)$ \\
\hline & Alcohol history & \\
\hline & None & $7(17.5 \%)$ \\
\hline & Light & $15(37.5 \%)$ \\
\hline & Moderate & $7(17.5 \%)$ \\
\hline & Heavy & $6(15.0 \%)$ \\
\hline & Unknown & $5(12.5 \%)$ \\
\hline & Primary site & \\
\hline & Tonsil & $24(60.0 \%)$ \\
\hline & Base of tongue & $16(40.0 \%)$ \\
\hline \multirow{7}{*}{ 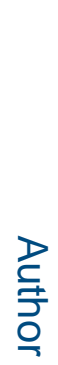 } & T-classification & \\
\hline & $\mathrm{T} 1$ & $15(37.5 \%)$ \\
\hline & $\mathrm{T} 2$ & $15(37.5 \%)$ \\
\hline & $\mathrm{T} 3$ & $4(10.0 \%)$ \\
\hline & $\mathrm{T} 4$ & $6(15.0 \%)$ \\
\hline & N-classification & \\
\hline & No & $1(2.5 \%)$ \\
\hline \multirow{2}{*}{ 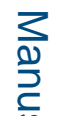 } & N1 & $5(12.5 \%)$ \\
\hline & $\mathrm{N} 2 \mathrm{a}$ & $7(17.5 \%)$ \\
\hline
\end{tabular}




\begin{tabular}{|c|c|c|}
\hline & Age at Enrollment, years (SD) & $60.3(8.5)$ \\
\hline & $\mathrm{N} 2 \mathrm{~b}$ & $19(47.5 \%$ \\
\hline & $\mathrm{N} 2 \mathrm{c}$ & $7(17.5 \%)$ \\
\hline & N3 & $1(2.5 \%)$ \\
\hline & Radiation dose & \\
\hline & $54 \mathrm{~Gy}$ & $25(62.5 \%$ \\
\hline & 60 Gy & $15(37.5 \%$ \\
\hline
\end{tabular}




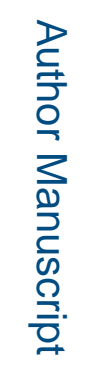

\begin{tabular}{|c|c|c|c|c|c|c|c|}
\hline 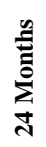 & 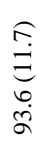 & $\frac{0}{0}$ & 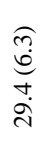 & $\stackrel{n}{?}$ & 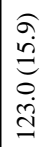 & 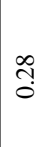 & $\frac{0}{i}$ \\
\hline$\sum_{\infty}^{\infty}$ & 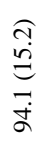 & $\begin{array}{l}\text { fo } \\
\text { Oे }\end{array}$ & $\begin{array}{l}\hat{\sigma} \\
\dot{e} \\
\overrightarrow{0} \\
0\end{array}$ & $\begin{array}{l}\mathscr{O} \\
\stackrel{0}{0} \\
0\end{array}$ & 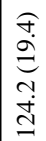 & 守 & $\begin{array}{l}\stackrel{\circ}{\sim} \\
\ddot{\sim}\end{array}$ \\
\hline 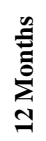 & 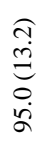 & $\begin{array}{l}\text { ஜூ } \\
8 \\
0\end{array}$ & 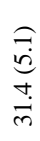 & ñ? & 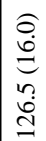 & 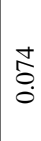 & $\begin{array}{l}0 \\
0 \\
\text { in }\end{array}$ \\
\hline
\end{tabular}

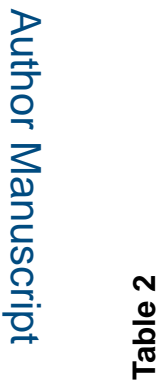

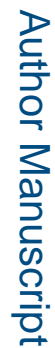

\begin{tabular}{|c|c|c|c|c|c|c|c|}
\hline 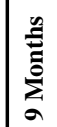 & 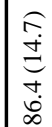 & $\stackrel{8}{8}$ & 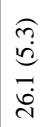 & $\stackrel{g}{0}$ & 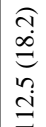 & $\stackrel{n}{0}$ & $\mid \begin{array}{l}0 \\
0 \\
0 \\
i\end{array}$ \\
\hline
\end{tabular}

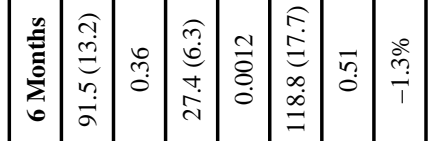

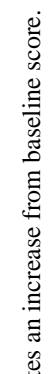

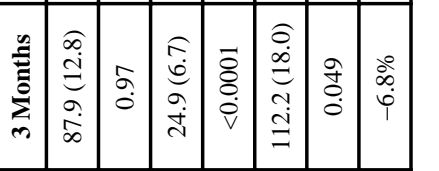

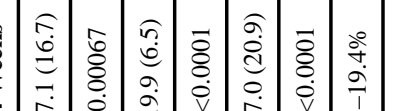

\begin{tabular}{|c|c|c|c|c|c|c|c|c|}
\hline 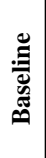 & 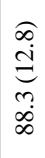 & I & 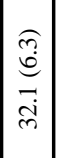 & I & 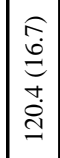 & 1 & 1 & \\
\hline & 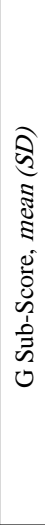 & 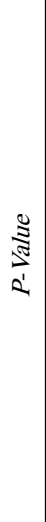 & 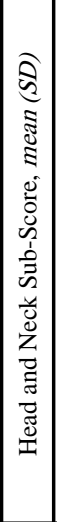 & 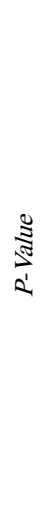 & 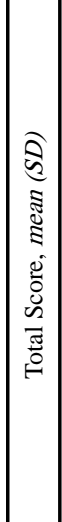 & 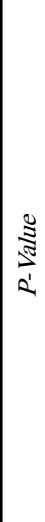 & 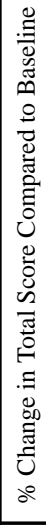 & \\
\hline
\end{tabular}

Cancer. Author manuscript; available in PMC 2018 April 25. 